\title{
LA FUNCIÓN TEXTUAL DEL ESPACIO TOPOGRÁFICO EN THE PLUMED SERPENT, DE D. H. LAWRENCE
}

\section{THE TEXTUAL FUNCTION OF TOPOGRAPHIC SPACE IN D.H. LAWRENCE'S THE PLUMED SERPENT}

\author{
Nora Marisa LEÓN-REAL MÉNDEZ \\ Instituto Tecnológico y de Estudios Superiores de Monterrey (ITESM) \\ nora.marisa@itesm.mx
}

Resumen: En este trabajo se presenta una lectura de la novela The Plumed Serpent (1926), de D. H. Lawrence, a partir de la construcción de significado del espacio mexicano en la narración. En esta obra, las descripciones del espacio topográfico no se limitan a mostrar un escenario de trasfondo. Por el contrario, Lawrence inviste de una carga significativa a las marcas geográficas, construyendo un espacio narrativo propio a la vez que ligado con el México fuera del texto. La descripción del paisaje sirve como método de creación de mundo, como técnica de formación del espacio narrativo.

Abstract: In this essay I present a reading of D.H. Lawrence's The Plumed Serpent (1926) through the process of making meaning of Mexican space. In this novel, descriptions of topographic space are not limited to showing a background setting. On the contrary, Lawrence confers significant meaning to the landmarks, constructing a narrative space that is particular as well as 
linked to the Mexico outside the text. Landscape description is used then as a method for world-making, as a technique for creating narrative space.

Palabras clave: Literatura inglesa. The Plumed Serpent. D. H. Lawrence. Espacio narrativo. México. Siglo veinte.

Key words: English Literature. The Plumed Serpent. D. H. Lawrence. Narrative Space. Mexico. Twentieth Century.

Las décadas de 1920 y 1930 constituyeron el epítome de la experiencia literaria inglesa fuera del Imperio Británico en el siglo Xx. Tras las aventuras del siglo XIX narradas por autores como Rudyard Kipling o Rider Haggard, el periodo entre las dos grandes guerras mundiales quedó marcado por los viajes realizados por los escritores ingleses fuera de su centro sociocultural. Así, estas décadas se destacan no solamente por la publicación de novelas ubicadas en países lejanos y desconocidos para los lectores ingleses, sino también por la consolidación del concepto del autor-viajero que observa de primera mano los universos que anteriormente eran descritos únicamente por exploradores y conquistadores. A partir de ese momento, los territorios menos conocidos del mapa están al alcance de individuos que, ya sea por el horror causado por la Gran Guerra y la inminencia de un segundo enfrentamiento bélico, o porque han dejado de encontrar en los tópicos de la cultura europea una fuente auténtica para explorar sus intereses literarios, deciden salir del universo cómodo y conocido del Imperio Británico y sus países vecinos para internarse en el Nuevo Mundo.

Es en este contexto que, según apunta Paul Fussell en su libro Abroad, D. H. Lawrence «can be seen as the vanguard of the British Literary Diaspora» (1980: 11), seguido casi de inmediato por otros escritores ingleses entre los que se encuentran Aldous Huxley, Evelyn Waugh, Graham Greene, Malcolm Lowry, Ralph Bates y Arthur Calder-Marshall. A pesar de su precaria salud, Lawrence conoce y escribe sobre cuatro continentes: Europa, África, Oceanía y, finalmente, América. Para él como para ningún otro, la «sensación de lugar» se vuelve una idea elusiva que debe ser perseguida como una premisa esencial para la creación literaria. Más allá de las ideas, para Lawrence es necesario encontrar el espacio en el que estas ideas son válidas, y el contexto social inglés le parece más que opresivo, sofocante. Es por ello que Lawrence busca en otros espacios la sensación de lugar apta para escribir, y esto le lleva, en 1923, a México.

Junto al Lago de Chapala, en Jalisco, Lawrence encuentra el lugar apropiado para situar una novela, que se publica en 1926, bajo el título de The 
Plumed Serpent, sobre la vida espiritual de Méxicoํ․ Este país nunca ha formado parte del Imperio Británico, y se encuentra dentro del imaginario de la cultura inglesa principalmente a través de narraciones de fallidos intentos de exploración y conquista. Pero, a partir de la obra de Lawrence, el país se instala en las letras inglesas dentro del género de la novela. La obra fundacional de este autor marca el primero de varios esfuerzos por utilizar México como escenario de un debate espiritual que no tiene cabida en las islas británicas. En conjunto, Lawrence y quienes le siguieron formaron un corpus de literatura inglesa ubicada en México con el cual crearon un espacio narrativo, una construcción retórica de referencias topográficas que remiten al México que los propios autores han observado en sus viajes por el país.

La búsqueda del lugar apropiado para la creación literaria se convirtió en tal grado en una obsesión para Lawrence que el autoexilio fue su forma de vida, especialmente en los últimos años. Incluso en sus primeras novelas, situadas todavía en Inglaterra, existe ya lo que John Worthen define en $D$. $H$. Lawrence: The Life of an Outsider como «awareness of the landscape» (2005: 30). Para el momento de su viaje a México, esta conciencia del paisaje se ha transformado ya, según afirma Ronald Walker en Infernal Paradise: Mexico and the Modern English Novel, en «dependence upon place to embody his own 'quests'» (1978: 103), hasta el punto de que en la posteridad se reconoce a Lawrence por «his acute, almost neurotic, sense of place» (Fussell, 1980: 145). El estilo de Lawrence está marcado por la importancia que tiene el lugar en sus obras, y el impacto que causa - la potente influencia del lugar en la trama - tiene su origen en el esfuerzo realizado por el autor en situarse a sí mismo en los espacios indicados para la creación del ambiente que busca en sus novelas.

Aunque Lawrence hereda el bagaje literario del Imperio Británico, él decide buscar sus propios referentes espaciales de forma directa viajando a lugares que, si bien no son completamente desconocidos en la literatura inglesa, no son tampoco clichés literarios del canon de su época. Así, Lawrence empieza recorriendo la campiña inglesa para salir luego de la isla rumbo al sur de Europa, en Italia, y embarcarse rumbo a África, Australia y, luego de años de planeación, a América. De acuerdo con Worthen, «New Mexico turned out to be the place Lawrence had been looking for all his life» (2005: 273). Pero, a pesar de que el rancho en Taos se convertiría en el lugar ideal en su

1 Lawrence había ya encontrado esta «sensación de lugar» cerca del agua, escribiendo Sons and Lovers, junto al Lago di Garda en Italia; «The Sisters», junto al mar de Fiascherino; Women in Love, en la costa de Cornwall, y Kangaroo en el Pacífico australiano (Worthen, 2005: 288). 
memoria, no es ahí donde decide situar su única novela en espacio americano. No es sino hasta que cruza la frontera hacia lo que él mismo llamaba el Viejo México que encuentra el lugar ideal para la escritura. Walken justifica esta preferencia debido a que «Mexico was much more compelling as a symbol of psychic revolution» (2005: 77). La idea precede al espacio - Lawrence tenía ya en mente una obra sobre la revolución espiritual, por lo que se lanza en busca de un lugar apto para esta-, pero sin él, la novela es imposible. Con esta idea sobre lo que debía ser su nueva obra, «Lawrence had needed Chapala - with its frequent fiestas, its abandoned villas and hotels, its quaint church (Iglesia de la Parróquia [sic]) and especially the lake - a symbolic setting» (Walken, 2005: 59). La historia puede existir una vez que Lawrence ha encontrado el lugar apropiado para ella. El siguiente paso es darle forma en su obra a través de su representación narrativa.

El espacio narrativo mexicano se construye en The Plumed Serpent a partir de la descripción de los elementos geográficos ineludibles del paisaje que se incluyen como marca de que la novela sucede en este país y en ningún otro. Estos elementos son tanto demarcaciones geográficas naturales - lagos, costas, barrancas, montañas, volcanes - como construcciones comunitarias que se han creado alrededor de ellas — villas, pueblos, ciudades-, partiendo de la premisa de que el escenario en el que se ubican es un espacio nuevo para la mayoría de los lectores que el propio autor tenía en mente en el momento de escribir su obra. Desde este ángulo de análisis, el paisaje es un concepto que permite analizar el espacio como parte de la construcción retórica que es la novela. El paisaje narrado, una imagen descrita en palabras, remite a algo observable desde un punto fuera del mismo creando un fuerte impacto visual. Y es que, desde la inmensidad del país que visita Lawrence, la descripción del paisaje que va a utilizar en su novela es la técnica más efectiva de darle una forma concreta al espacio en blanco, vacío de significado para muchos lectores.

La descripción del paisaje convierte a México en una unidad diegética funcional dentro de sus obras: el espacio mexicano. De acuerdo con Ronald Barthes, en su introducción al Análisis estructural del relato, la función o la cualidad funcional es una de las unidades narrativas mínimas que cargan de sentido al relato (1996: 12). Así, cuando me refiero al espacio mexicano como funcional, argumento que no se trata solo de un trasfondo, sino de una unidad significativa «que se present[a] como el término de una correlación [...] que madurará más tarde al mismo nivel» (Barthes, 1996: 12). Al evocar ciertas marcas del paisaje relevantes en la novela - el Lago de Chapala, el volcán Popocatépetl- Lawrence busca provocar en los lectores una «sensa- 
ción de lugar», colocando al espacio en un nivel asequible, comprensible y, entonces, apto para la narración, funcional al nivel de la trama. El espacio topográfico no se limita entonces al nivel de escenario, de trasfondo de la acción. El paisaje mexicano no tiene únicamente una función decorativa y su presencia no indica solamente la posición geográfica de los acontecimientos. Por el contrario, el análisis de los procesos de representación deja ver que se inviste de una carga altamente significativa a las marcas geográficas del espacio topográfico. Pero primeramente la descripción del paisaje sirve como método de creación de mundo, como técnica de formación del espacio como concepto básico narrativo.

En este ensayo se analizan dos de los mecanismos retóricos utilizados por Lawrence con este propósito: la nominación y el contraste. Mediante el uso de estos dos tropos, el autor es capaz de crear un mundo intratextual que surge de su propia experiencia en México. Así, la nominación de marcas topográficas en el territorio mexicano funciona como una liga entre el mundo interno de su novela y el México que él mismo ha visitado, que sus lectores pueden reconocer ya sea por viajes propios o por la simple consulta de un mapa o un libro de viaje $^{2}$. Por otra parte, la negación de nombrar ciertas marcas por su nombre real, asignándoles un nombre alterno, nos indica ya la intención del autor de crear un universo coherente consigo mismo y no como una copia exacta del México que él conoce. Esto se revela también en el contraste realizado en la novela entre dos tipos de espacio topográfico: el espacio urbano y el rural. Aunque el contraste se realiza entre dos espacios definidos por cualidades topográficas, la importancia de este tiene una tonalidad ideológica que debe entenderse como parte del universo intratextual creado por Lawrence. La diferencia entre ambos implica la superioridad de uno sobre otro que debe entenderse en el contexto interno de la obra. El estudio de estos dos métodos nos permite poner en evidencia la conformación retórica del espacio mexicano en esta obra que se convertirá luego en punto de partida para las novelas posteriores escritas por los autores de la diáspora inglesa en México.

\section{DE LA CIUDAD DE MÉXICO A SAYULA. LA NOMINACIÓN COMO ÍNDICE DE REALIDAD}

The Plumed Serpent se abre con una frase que sitúa la novela de forma espacio-temporal: «It was the Sunday after Easter, and the last bull-fight of

\footnotetext{
2 Entre estos se pueden incluir sus propios relatos de viaje compilados en Mornings in Mexico.
} 
the season in Mexico City» (Lawrence, 1995: 3). Antes de presentar a los personajes o al conflicto con el que arranca la trama, la narración nos ubica ya en el momento y el lugar de la acción. Sin necesidad de incluir coordenadas para su ubicación, la obra de Lawrence utiliza únicamente el nombre de la ciudad: Mexico City. Este es suficiente para que los lectores comprendan a qué espacio se refiere la narración, incluso si no han estado nunca en él, porque forma parte de un conocimiento cultural común.

La utilización del nombre de la ciudad en esta primera frase es un indicio de la importancia que el espacio tiene en la novela. Más allá de la descripción minuciosa de la Ciudad de México, el hecho de que sea simplemente nombrada, sin adjetivos, habla ya de las expectativas de la narración: se espera que el lector entienda a qué se refiere el nombre sin necesidad de ser aclarado. El uso del nombre propio funciona en casos como este, de acuerdo con Carrol Clarkson, en «Remains of the Name», «to mark sites of human significance on indifferent ground. In an inversion of the expected sequence - first the landscape, then the name - we can say that a landscape becomes literary (which is to say, it is given written form) precisely because it is named» (2008: 135). La Ciudad de México es un espacio significativo para la acción, cuya importancia podemos deducir del uso deliberado de su nombre. La novela abre con un espacio conocido para orientar a los lectores en el marco extratextual antes de darle un significado interno dentro de la obra.

De acuerdo con Luz Aurora Pimentel, en El espacio en la ficción, la utilización de nombres propios que coinciden con la realidad extratextual tiene la función de facilitar la ubicación del lector en los hechos de la trama. Para Pimentel, «la nomenclatura, de entrada, le ofrece al lector una ilusión de realidad 'autorizada' por un referente 'real' fuera del texto y/o por una realidad 'compartida' que solo hay que reconocer» (2001: 26, énfasis original). En el caso de The Plumed Serpent, esta autorización es facilitada por el nombre de la Ciudad de México acompañado de otros referentes espaciales que ayuden al reconocimiento del lugar. Así, el narrador nos indica que Kate Leslie, la protagonista, «had been in many cities of the world, but Mexico had an underlying ugliness, a sort of squalid evil, which made Naples seem debonair in comparison» (Lawrence, 1995: 16). México no requiere ya el apelativo de «City» porque la frase descriptiva nos indica que se refiere a la ciudad y no al país (homónimo) y lo enfatiza al compararla con otra ciudad, Nápoles. Pero, además de explicar el tipo de espacio al que se refiere, en esta oración el narrador hace uso también de una variable comparativa, la fealdad de ambas ciudades, lo que permite colocarlas en la misma escala. 
Así, el lector que no conoce el espacio al que se refiere el nombre propio Mexico City, pero que conoce fácilmente el de Naples, puede reconocer las características del espacio al que se refiere el narrador por medio de la comparación.

La presentación del espacio depende de este reconocimiento más que la noción estética del lugar. La narración presupone del lector también un conocimiento del estilo de vida de la Ciudad de México cuando uno de los personajes afirma que ha imaginado «all the things that are supposed to happen in Mexico - chauffeur driving away with you into some horrible remote region, and robbing you and all that» (Lawrence, 1995: 19). Con ello, la novela no solo asume sino que confirma una noción característica del espacio nombrado como Ciudad de México como un lugar, en este caso, peligroso. La equiparación entre el espacio en el que sucede la acción, en el que se mueven los personajes, y la Ciudad de México visitada por Lawrence entre 1923 y 1925, surge del hecho de que el narrador usa el nombre real de la ciudad en la novela. Así, de acuerdo con Pimentel, «[n]ombrar es conjurar. De todos los elementos lingüísticos que se reúnen para crear una ilusión de realidad, el nombre propio es quizás el de más alto valor referencial [...] Así, dar a una entidad diegética el mismo nombre que ya ostenta un lugar en el mundo real es remitir al lector, sin ninguna otra mediación, a ese espacio designado y no a otro» (2001: 29). En The Plumed Serpent no existe entonces ningún otro posible referente. El reconocimiento de lo que significa «Mexico City» permite al lector la comprensión de las acciones en los primeros capítulos de la novela, marcando con ello un paralelismo entre las acciones ficticias de la obra con lo que potencialmente sucede realmente en la capital mexicana.

Al analizar el uso de nombres propios en la descripción del espacio narrativo, Pimentel encuentra que, especialmente en el caso de nombres de ciudades, su uso es más que una referencia geográfica, pues se convierte en una forma de transportar por entero un concepto del mundo real al interior del texto literario - incluyendo características físicas e ideológicas-. Así, «el nombre de una ciudad en un texto narrativo remite a un lugar individual, localizable en la geografía del mundo; que, al mismo tiempo, la ciudad de ficción establece relaciones intertextuales con otros discursos que han dado ‘cuerpo semántico' a la entidad real» (Pimentel, 2001: 33). Lawrence hace uso de esta técnica más de una vez. Además de los ejemplos vistos sobre la capital mexicana, en el segundo capítulo alude también al puerto principal 
de entrada para los visitantes europeos: Veracruz ${ }^{3}$. Así, aunque Kate - como el propio Lawrence - ha llegado en tren desde Estados Unidos, su primo Owen «had come through Vera Cruz, and had had his fright; the porters had charged him twenty pesos to carry his trunk from the ship to the train» (Lawrence, 1995: 30). De nuevo, sin hacer uso de otro medio de descripción del espacio además del nombre, se da por entendido que el Veracruz del texto es una puerta de entrada al país que tiene las mismas características que el puerto mexicano. Cualquier inglés que buscara entrada a México tendría que saber de la existencia de esta ciudad, pues aparece no solo en el boleto del barco, sino en la literatura de exploración y viajes previa a la llegada de Lawrence al país. Al mencionar su nombre, la novela establece relaciones fuera del texto para componer el sentido de este espacio dentro de la obra.

Esta técnica de nominación pura, que excluye la descripción del espacio más allá de su nombre, tiene como consecuencia que el lector cree una correspondencia entre el espacio de ficción y el espacio geográfico de México. Como afirma Pimentel, «cuando la ciudad ficcional no se describe sino solo se nombra, mañosamente se la declara idéntica a la ciudad real» (2001: 43). Así, la Ciudad de México en la que sucede la corrida de toros del primer capítulo, y el puerto de Veracruz por el que hace su entrada en el país Owen, deben entenderse como desdoblamientos de la capital y del puerto de la República Mexicana tal como existían en la década de 1920. Lo mismo sucede con otros espacios nombrados dentro de la Ciudad y reconocibles fácilmente en cualquier guía para turistas: el Museo Nacional, el Zócalo, la Catedral y la Universidad Nacional, incluyendo los frescos pintados por Diego Rivera. Su función dentro de la trama es fácilmente comprensible con el simple uso de su nombre porque deben ser entendidos como idénticos a los edificios homónimos en la Ciudad de México.

Como método de descripción narrativa, la nominación de un espacio real es uno de los más efectivos y económicos porque con muy pocas palabras -incluso una - logra integrar en el mundo ficcional toda una realidad extratextual. Pimentel afirma que «nombrar una ciudad, aun sin describirla, es suficiente para proyectar un espacio ficcional concreto, ya

3 La ortografía de la palabra ha cambiado con el paso del tiempo. Aunque el nombre completo de la ciudad en el momento de su fundación era Villa Rica de la Vera Cruz, ya había sido reducido coloquialmente a Vera Cruz en el momento en que los autores ingleses viajaron a México. Actualmente, el nombre oficial se ha consolidado como Veracruz. Utilizo Vera Cruz cuando cito directamente de los textos, y Veracruz como parte de mi propio discurso. 
que el nombre propio es, en sí mismo, una descripción en potencia» (2001: 32). Michel Foucault analiza esta idea en Las palabras y las cosas, pero él dirige su atención a la consecuencia insidiosa de la técnica cuando indica que «el nombre propio no es más que un artificio: permite señalar con el dedo, es decir, pasar subrepticiamente del espacio del que se habla al espacio que se contempla, es decir, encerrarlos uno en otro con toda comodidad, como si fueran mutuamente adecuados» (2004: 19). Para evitar esta generalización entre las cosas — provocada por las palabrasFoucault propone que «si se quiere mantener abierta la relación entre el lenguaje y lo visible, si se quiere hablar no en contra de su incompatibilidad sino a partir de ella, de tal modo que se quede lo más cerca posible del uno y del otro, es necesario borrar los nombres propios y mantenerse en lo infinito de la tarea» (2004: 19). Lo que The Plumed Serpent logra en los primeros capítulos es precisamente cerrar la relación entre el lenguaje y lo visible, convertirlo en lo mismo para facilitar la inmersión del lector en el texto. La novela de Lawrence no solo no deja de lado el uso del nombre propio, sino que se aprovecha de la carga semántica que estos nombres tienen ya en el saber popular para la construcción del espacio mexicano hasta el punto de hacerlo parecer idéntico a México.

Pero más allá de realizar un paralelismo visual entre el espacio real y el narrativo, el uso del nombre propio describe cualitativamente el espacio, puesto que «el nombre que lo designa no solo tiene un referente sino un sentido, ya que, precisamente por ser un espacio construido, está cargado de significaciones que la colectividad/autor(a) le ha ido atribuyendo gradualmente» (Foucault, 2004: 31). Una vez que el lector está ubicado en el espacio, gracias al nombre, el narrador de The Plumed Serpent procede a describirlo en el tercer capítulo:

Superficially, Mexico might be all right: with its suburbs of villas, its central fine streets, its thousand of motorcars, its tennis, and its bridgeparties. The sun alone shone brilliantly every day, and big bright flowers stood out form the trees. It was a holiday.

Until you were alone with it. And then the undertone was like the low, angry, snarling purring of some jaguar spotted with night. There was a ponderous, down-pressing weight upon the spirit: the great folds of the dragon of the Aztecs, the dragon of the Toltecs winding around one and weighing down the soul. And on the bright sunshine was a dark steam of an angry, impotent blood, and the flowers seem to have their roots in spilt blood. The spirit of place was cruel, down-dragging, destructive (Lawrence, 1995: 42). 
La descripción, que empieza aludiendo al espacio físico — las villas, las calles, las flores-, se concentra en el siguiente párrafo en la sensación provocada por este espacio. Y, marcando una contradicción que permanece a lo largo de toda la obra, la belleza física del espacio no corresponde con el impacto que causa en quienes están en él, sino que tiene el efecto contrario. Es por ello que, mientras que para darle forma física al espacio se recurre al conocimiento colectivo de México, para la construcción del significado que este tiene en los personajes sí es necesaria la descripción más allá del nombre. Aunque la novela parte de la referencia extratextual con el país, poco a poco construye una realidad intratextual mediante la carga de significaciones que se agregan en la construcción narrativa del espacio mexicano.

Una vez que la protagonista abandona la Ciudad de México y se dirige a Sayula, las referencias extratextuales mediante el uso de los nombres propios disminuyen considerablemente, pues Lawrence decide cambiar los nombres de los lugares visitados por él mismo - los que corresponden al mapa mexicano- en los siguientes capítulos de su obra. En El relato en perspectiva, Luz Aurora Pimentel encuentra que «[e]xisten descripciones que deliberadamente subvierten la ilusión referencial, aunque curiosamente a partir de los mismos elementos léxico-sintácticos antes mencionados» (1998: 37). Así, Chapala - tanto el lago como el pueblo del mismo nombre - aparecen en la novela bajo el nombre de Sayula, dándonos ya una primera marca de que el espacio mexicano de Lawrence es una creación propia, necesaria para el desarrollo de la trama. El nombre de Sayula aparece por primera vez en la novela en la noticia del periódico leída por Kate que le introduce en el conflicto religioso alrededor del que girará la trama. La nota (como la novela misma) sitúa la narración en el espacio desde la primera línea: «There was a ferment in the village of Sayula, Jalisco, on the Lake of Sayula» (Lawrence, 1995: 48). Existe aquí un juego entre el espacio extratextual y el construido para fines narrativos. Al mencionar el estado de Jalisco —en el que se encuentra Chapala—, así como su posición en el lago del mismo nombre, Lawrence crea una relación referencial entre el espacio narrativo y el real. Pero él mismo subvierte este índice de realidad al modificar el nombre más importante. Se crea entonces una relación de similitud entre ambos espacios - intra y extratextual-, pero se marca también una distancia clara entre ambos. Una vez que se ha introducido a los personajes dentro del país a través de vías realistas - correspondientes con la posible experiencia de un viajero inglés que entra a México en la década de los 20la narración procede a distinguir el espacio mexicano creado por el autor del México que ha visitado. 
Vemos entonces en The Plumed Serpent un proceso que empieza haciendo uso de México como referencia espacial que liga la credibilidad de la narración —su verosimilitud - al mundo real, puesto que «la referencia extratextual es garantía de 'realidad'» (Pimentel, 1998: 31). Pero, gradualmente, estas referencias van ganando significado intratextual. Más allá de la primera línea, que se reduce a mencionar únicamente el nombre del espacio, la repetición del nombre, ligada a una serie de descripciones físicas y calificativas, crea una imagen dentro del texto de lo que significa Mexico City más allá de la referencia con la capital mexicana. Todavía más importante es la repetición del nombre en el caso de Sayula. Dado que no existe una concordancia «perfectamente sinecdótica» (Pimentel, 1998: 31) que refiera a Sayula con su correlato en el mapa de México, es solamente a través de la construcción intratextual que se podría crear esta conexión. Por eso, para Pimentel, aunque «se insiste en la referencia extratextual y el mito compartido, la referencia intratextual garantiza la coherencia, legibilidad y 'universalidad' de su construcción verbal» (1998: 32). La concordancia entre el nombre de los lugares dentro del texto y los lugares reales de México tiene una primera función: dar autoridad a la narración para hablar de un espacio específico, lejano y desconocido. Pero, narrativamente, es mucho más importante que este espacio tenga un significado interno para la trama, por lo que es por medio de la descripción repetida y coherente de los lugares - en este caso, mediante el uso de su nombre propio, aunque sea distinto al del mundo extratextual - que se crea el espacio mexicano.

Existe entonces un doble proceso de referencialidad: uno que remite al mundo fuera del texto y otro que se asegura que el espacio interno tenga sentido. Según Pimentel en El espacio en la ficción, es gracias a este doble fenómeno que «es posible construir un 'mundo' de ficción capaz de establecer relaciones significantes, de concordancia o de discordancia, con el mundo real» (2001: 38-39). Por una parte, el uso de nombres con referentes extratextuales asume una concordancia entre el texto y el mundo que puede explicarse, de acuerdo con Foucault, porque «a fin de que sea posible un nombre común, es necesario que haya entre las cosas esta semejanza inmediata que permita a los elementos significantes el correr a lo largo de las representaciones, el deslizarse por su superficie, el asirse a sus similitudes para formar, por último, designaciones colectivas» (2004: 146). El uso de la misma palabra implica que las cosas son, hasta cierto punto, las mismas. Pero existe también una necesidad narrativa de ir más allá de la referencia, de construir un universo coherente que funcione en concordancia con las acciones de los personajes. Para alcanzar esta coherencia interna la narra- 
ción no requiere un referente en el mundo externo, sino una consistencia hacia dentro del texto. Es este segundo proceso el que no solo permite sino que enriquece la novela con la creación de un Sayula dentro de México. Así, aunque en una primera instancia el espacio mexicano surge en The Plumed Serpent bajo el cobijo de la correlación con el México real, su construcción se consolida gradualmente mediante un proceso de autorreferencialidad. En ambos casos, el uso del nombre propio es una herramienta primordial para la creación del espacio narrativo.

\section{LA CIUDAD FRENTE AL CAMPO. EL CONTRASTE COMO MÉTODO DE EXPOSICIÓN IDEOLÓGICA}

Si bien la nominación es la técnica más económica de construcción de espacio en la narración, pronto las obras recurren a otros mecanismos que no solo permiten conocer el mundo de la novela, sino que presentan dicho espacio desde una perspectiva personalizada, ya sea por el narrador o por los propios personajes. Mieke Bal (1990) en Teoría de la narrativa y Luz Aurora Pimentel en El espacio en la ficción coinciden en que el uso de modelos binarios contribuye a la significación de los espacios en el contexto narrativo. Pimentel afirma que, «[p]ara la proyección del espacio diegético y de los objetos que en él se distribuyen se recurrirá, necesariamente, a modelos binarios de espacialidad basados en categorías lógico-lingüísticas tales como cercano/lejano, arriba/abajo, pequeño/grande, vertical/horizontal, dentro/fuera, frente a/detrás de, etcétera» (2001: 60). El contraste es entonces una técnica básica para la construcción de un mundo que tiene un significado que impacta en la trama de la novela. Mientras que la nominación cumple la función de construir un mundo diegético, el contraste entre dos conceptos espaciales permite infundir este mundo diegético con una significación ideológica.

Michel Foucault, quien como hemos visto encuentra en la nominación una forma problemática de significación, considera, al discutir los procesos de ordenación mediante los cuales se entiende el significado de las cosas, que la identidad de ellas «no consistirá ya en relacionar las cosas entre sí, a partir de la búsqueda de todo aquello que puede revelarse en ellas como un parentesco, una pertinencia y una naturaleza secretamente compartida, sino por el contrario en discernir, es decir, en establecer las identidades y después la necesidad del paso a todos los grados que se alejan» (2004: 62). Así, el espacio mexicano en particular que se construye en The Plumed Serpent adquiere una identidad propia — más allá de la reproducción del México 
conocido por Lawrence- mediante el establecimiento de diferencias entre conceptos particulares. El contraste entre conceptos espaciales queda de manifiesto en la distinción clara y constante que se realiza entre el espacio urbano y el rural —ejemplificados en la Ciudad de México y en Sayula, respectivamente- para mostrar cómo más allá del nombre (que refiere o no al mundo extratextual) existe una serie de categorías descriptivas que caracterizan estos espacios como distintos desde un binarismo que obliga a leerlos como significativamente peores/mejores entre sí. Así, The Plumed Serpent no solamente describe un México narrativo, sino que presenta un sistema de ideas, una ideología según la cual algunos espacios son mejores o peores que otros.

Esta superioridad de un concepto sobre otro como resultado del contraste se desprende, de acuerdo con Foucault, de que «la emulación deja inertes, una frente a otra, las dos figuras reflejadas que opone. Sucede que una sea la más débil y acoja la fuerte influencia de la que se refleja en su espejo pasivo» (2004: 29). Helena Beristáin coincide en su Diccionario de retórica y poética al definir que «[e]ntre los miembros antitéticos suele haber coordinación, pero también puede darse la subordinación. Su contenido puede ofrecer un carácter adversativo o disyuntivo» (2000: 55). En The Plumed Serpent se presenta una antítesis espacial en la cual hay una selección de un tipo de espacio sobre otro, una subordinación. En el momento en el que Kate, la protagonista, afirma que «[s] he wanted to leave the city» (Lawrence, 1995: 71), toma una decisión que implica abandonar el espacio urbano para internarse en el mundo rural, marcando una preferencia del segundo sobre el primero. Esta postura antitética se presenta, por ejemplo, en la descripción que hace Kate de la Ciudad de México y de Sayula durante la noche. En el segundo capítulo, la protagonista pasa una noche insomne en la capital mexicana, indicando que «through the night she could not sleep, but lay listening to the noises of Mexico City, then to the silence and the strange, grisly fear that so often creeps out on the darkness of a Mexican night. Away inside her, she loathed Mexico City. She even feared it. In the daytime it had a certain spell - but at night, the underneath grisliness and evil came forth» (Lawrence, 1995: 23). En absoluto contraste, el capítulo 16 nos presenta a la protagonista en Sayula, caminando junto al lago, donde exclama: «'But the night is beautiful,' said Kate, breathing deep» (Lawrence, 1995: 211). Tomando en cuenta que la base léxica de la antítesis son los antónimos (Beristain, 2000: 55), estos fragmentos describen antitéticamente el espacio nocturno urbano como horripilante («grisly»), en oposición al espacio nocturno rural como hermoso («beautiful»). La descripción de un 
mismo concepto mediante adjetivos tan opuestos presenta entonces un modelo binario en el cual un espacio se considera mejor que el otro, un mundo narrativo en el que el campo es un espacio más deseable que la ciudad.

Pero además de contribuir a la exposición de las ideas subyacentes en el texto - la perspectiva del narrador o los personajes sobre el espacio-, la presentación del espacio mexicano mediante el uso de descripciones contrastantes contribuye a crear un impacto específico en el lector. En su Diccionario de terminología literaria, Emma González de Gambier define el contraste como «la oposición o contraposición de actitudes, formas, contenidos que se establecen entre personas o cosas» y agrega que se trata de «un recurso estilístico que se emplea en la creación literaria para realzar la expresividad» (2002: 88). La representación de un paisaje, hecha desde la perspectiva dicotómica de un personaje protagonista, es más que una construcción en palabras de una imagen visual de dicho paisaje, sino que expresa una opinión particular del mismo. En otras palabras, se presenta ya con un código para la lectura. El estilo utilizado por Lawrence en esta novela enfatiza la diferencia entre el espacio y su impacto, como hemos visto, por la consciente distinción entre la descripción de lo visual y de la forma en que la imagen es percibida por el personaje. Es posible leer esta aparente paradoja en el texto desde la perspectiva de Beristáin cuando afirma que «[1]a yuxtaposición antitética de los términos refuerza sus significados, los aclara y los presenta con viveza» (2000: 56). La sensación del espacio mexicano en The Plumed Serpent surge del impacto causado en el lector por este contraste entre la descripción del espacio y los sentimientos que causa en los personajes.

Podemos también leer el desprecio que existe en esta novela por la Ciudad de México como epítome del espacio urbano, desde la perspectiva de Edward Said en Cultura e imperialismo, donde indica que «existe una creencia [...] obstinada de que las tareas coloniales de la metrópolis eran marginales y quizá hasta ajenas a las actividades culturales de las grandes culturas metropolitanas» (1996: 79). Dado que el propósito de la presencia de Kate en México es precisamente el deseo de no estar en las ciudades europeas en el momento posterior a la Gran Guerra, su postura ante otro espacio metropolitano - además, inferior, puesto que es una copia de los espacios de los colonizadores- causa un efecto de rechazo en ella. Así, cuando observa desde el techo de su hotel «the rough glare of uneven flat roofs, with loose telephone wires trailing across, and the sudden, deep, dark wells of the patios, showing flowers blooming in the shade» (Lawrence, 1995: 42, énfasis original), ella misma descubre que «[i]t ought to have been al gay, 
allegro, allegretto, in the sparke of brigh air and old roof surfaces. But no! There was the dark undertone, the black serpent-like fatality all the time» (Lawrence, 1995: 43, énfasis original). El efecto grotesco de los techos disparejos, los cables de teléfono desordenados y las flores que surgen de los pozos oscuros de los patios crea una imagen urbana que exalta precisamente el aspecto negativo de la vida en la ciudad y la impulsa a dirigirse a la extensión rural de México.

Como método de construcción de un mundo narrativo, el espacio mexicano, la descripción de la Ciudad de México tiene la característica de estar sujeta a modelos previos en la mente de los lectores. La permanencia de la protagonista en la capital mexicana dirigiría la narración en torno a estos modelos, puesto que «la descripción crea una ilusión de realidad gracias a que se adecua, no a la realidad, sino a los modelos de realidad construidos por otros discursos y que influyen en nuestra percepción del mundo conformándola a aquéllos» (Pimentel, 2001: 69, énfasis original). El traslado de la ciudad al campo es también un esfuerzo de abrir el universo de las posibilidades narrativas en la obra. El espacio metropolitano de la Ciudad de México no resulta verosímil - como capital de un país sumamente centralizado- - si el propósito es poner en escena una rebelión oculta de la que debe surgir el renacimiento espiritual de los mexicanos. El espacio rural ofrece aquí un doble efecto de libertad de movimiento. Dentro de la trama, los personajes pueden organizarse fuera de las fuerzas de control del Estado, centradas en la capital. Pero además, en el contexto narrativo, el autor puede construir un mundo sin las constricciones del modelo preconcebido en la mente de sus lectores.

Al abandonar la Ciudad de México para trasladarse a Sayula, la novela crea un espacio mexicano propio dentro del contexto verosímil de México. Este espacio surge en contraposición al espacio anterior, la ciudad, y su significación surge precisamente de la antítesis creada entre ambos. La línea del paisaje en el campo no ofrece techos monstruosos sino «the steep slope of the hills and the bit of flat by the lake» (Lawrence, 1995: 143). La llegada a Sayula permite la creación de un espacio significativo para la trama y no previamente significado en el contexto cultural de la época. Pero la significación asignada a este espacio como superior al anterior surge del contraste entre ambos, de relacionar el espacio urbano con lo grotesco y el rural con la tranquilidad del campo. Sayula es una expresión de la libertad creativa ejercida en la novela; la libertad de elegir un nombre y describirlo según las necesidades de la obra, y no las del mundo extratextual. Y el impacto de esta 
imagen, la sensación del lugar, surge del contraste presentado entre el espacio urbano y el rural, entre la Ciudad de México y Sayula.

\section{CONCLUSIONES}

Al analizar la representación del espacio mexicano en The Plumed Serpent hemos resaltado el uso de dos herramientas retóricas utilizadas por el autor para construir un espacio narrativo. En primera instancia, el hecho de que este punto geográfico corresponda con el espacio extratextual de México parece ser lo más destacable, lo que en mayor medida permite caracterizar y significar el escenario, pero pronto se vuelve necesaria también la creación de espacios propios a la narración. Así, aunque existen referencias al México fuera del texto, la novela ejerce también la libertad poética de su autor al no pretender presentar una reproducción mimética idéntica a este espacio extratextual. La nominación se convierte en la herramienta principal para la creación de mundo, y su contribución en la representación del espacio mexicano es la introducción de lugares con correspondientes en el mundo extratextual - entre los que destacan la Ciudad de México y el puerto de Veracruz- que marcan un índice de la realidad mexicana en el texto, pero también la conformación de sitios cuyo significado existe únicamente dentro de las obras, como en el caso de Sayula. Este último, aunque inspirado en una región dentro del territorio mexicano visitada por Lawrence - Chapala-, ha sido significativa y repetidamente nombrado de forma distinta para enfatizar que su función dentro de la novela es superior a su capacidad de remitir al lector a un espacio existente en México. El hecho de que en este lugar sucedan efectivamente las acciones más importantes de la obra nos indica que el autor ha superpuesto la importancia de la trama sobre la reproducción realista del paisaje de México.

Inspirado, entonces, pero no constreñido por el país, Lawrence crea su propio espacio mexicano bajo una premisa: que el lector debe entender que la historia sucede en México. El espacio narrativo debe ser creíble sin ser tan minucioso que quien no lo conozca sea incapaz de identificarlo en el texto. Además del uso del nombre propio, Lawrence utiliza otras herramientas que describen el espacio mexicano. El contraste ayuda a la representación de ciertos sitios al ponerlos en paralelo con otros, a los que se considera totalmente diferentes. Al incluir en el texto dos o más lugares existe una validación del espacio narrativo, consolidando el mundo del texto como universo posible. Más allá de la representación del espacio mexicano como un paisaje, una vista observable, esta novela privilegia la funcionalidad de 
dicho espacio para la trama. La experiencia vivida en un espacio va más allá de lo que las posibles obras hechas sobre este puedan representar. Tal como afirma Rick Van Noy, en Surveying the Interior: Literary Cartographers and the Sense of Place, «the challenge of knowing a place [involves] a more complex and thorough kind of environmental reading, the perceptual shift required to move from the abstract to the particular» (2003: 3). La representación del espacio topográfico implica un proceso de transición de lo netamente geográfico a lo significativo en términos narrativos, funcional para la trama. La representación del espacio mexicano implica entonces un proceso de construcción de mundo, más que de reproducción del mismo. Conforme la obra se escribe, se publica y es leída por otros autores ingleses interesados en México como locación, el corpus de literatura inglesa sobre el espacio mexicano crece y se alimenta de sí mismo. El espacio mexicano es por tanto uno de los universos narrativos de la literatura inglesa, un topos, consolidado en cada una de sus manifestaciones, pero inaugurado por D. H. Lawrence con la publicación de The Plumed Serpent.

\section{REFERENCIAS BIBLIOGRÁFICAS}

BAL, M. (1990). Teoría de la narrativa: Una introducción a la narratología. Madrid: Cátedra.

BARTHES, R. (1996). «Introducción». En Análisis estructural del relato, R. Barthes (ed.), 7-38. México: Ediciones Coyoacán.

BERISTÁIN, H. (2000). Diccionario de retórica y poética. México: Porrúa.

CLARKSON, C. «Remains of the Name». En Literary Landscapes: From Modernism to Postcolonialism, Attie de Lange et al. (eds.), 125-142. New York: Palgrave MacMillan.

FOUCAULT, M. (2004). Las palabras y las cosas. México: Siglo Veintiuno.

FUSSELL, P. (1980). Abroad: British Literary Traveling Between the Wars. New York: Oxford University Press.

GONZÁLEZ DE GAMBIER, E. (2002). Diccionario de terminología literaria. Madrid: Síntesis.

LAWRENCE, D. H. (1927). Mornings in Mexico. London: Martin Secker.

- (1995). The Plumed Serpent. Hertfordshire: Wordsworth.

PIMENTEL. L. A. (1998). El relato en perspectiva. Estudio de teoría narrativa. México: Siglo Veintiuno.

- (2001). El espacio en la ficción. México: Siglo Veintiuno. 
SAID, E. (1996). Cultura e imperialismo. Barcelona: Anagrama.

VAN NOY, R. (2003). Surveying the Interior: Literary Cartographers and the Sense of Place. Reno: University of Nevada Press.

WALKER, R. (1978). Infernal Paradise: Mexico and the Modern English Novel. Berkeley: University of California Press.

WORTHEN, J. (2005). D. H. Lawrence: The Life of an Outsider. New York: Counterpoint.

Recibido el 8 de abril de 2013.

Aceptado el 19 de septiembre de 2013. 